\title{
Infrared digital holography applications for virtual museums and diagnostics of cultural heritage
}

M. Paturzo

A. Pelagotti

A. Geltrude

M. Locatelli

P. Poggi

R. Meucci

P. Ferraro

E. Stoykova

F. Yaraş

A. Özgür Yontem

H. Kang

L. Onural 


\title{
Infrared digital holography applications for virtual museums and diagnostics of cultural heritage
}

\author{
M. Paturzo*a ${ }^{* a}$ A. Pelagotti ${ }^{\text {b }}$, A. Geltrude ${ }^{\text {b }}$, M. Locatelli $^{\text {b }}$, P. Poggi ${ }^{\text {b }}$, R. Meucci ${ }^{\text {b }}$, P. Ferraro ${ }^{\text {a }}$, E. \\ Stoykova $^{c, d}$, F. Yaraş ${ }^{d}$, A. Özgür Yontem ${ }^{d}$, H. Kang ${ }^{d}$, L. Onural ${ }^{d}$, \\ ${ }^{a}$ CNR -Istituto Nazionale di Ottica, via Campi Flegrei 34, 80078, Pozzuoli, Italy \\ ${ }^{\mathrm{b}}$ CNR -Istituto Nazionale di Ottica, L.go E. Fermi 6, 50125, Firenze, Italy; \\ ${ }^{c}$ Institute of optical materials and technologies - BAS, 1113 Sofia, Bulgaria \\ d Bilkent University, Department of Electrical and Electronics Engineering, TR-06800 Ankara, \\ Turkey.
}

\begin{abstract}
Infrared digital holograms of different statuettes are acquired. For each object, a sequence of holograms is recorded rotating the statuette with an angular step of few degrees. The holograms of the moving objects are used to compose dynamic 3D scenes that, then, are optically reconstructed by means of spatial light modulators (SLMs) using an illumination wavelength of $532 \mathrm{~nm}$. This kind of reconstruction allows to obtain a 3D imaging of the statuettes that could be exploited for virtual museums.
\end{abstract}

Keywords: Infrared display, digital holography.

\section{INTRODUCTION}

Infrared (IR) digital holograms have some advantages compared to those ones recorded with a visible radiation source. Firstly, thanks to the long wavelength used, the distance between the camera and the object can be reduced about a fourth with respect to that of visible holograms. Moreover, the high output power of IR laser sources and the lesser sensitivity to seismic noise, when compared to the setup exploiting the visible spectrum, make them suitable for the recording of large objects.

Such holograms can be optically reconstructed using a visible laser and a SLM device. Potential difficulties in the visible reconstruction of IR holograms could be caused by the possible optical aberrations involved. Generally, if a hologram is reconstructed with a wavelength different than the one used in the recording process, the resulting image is affected by some aberrations that depend on the wavelength ratio of reconstructing to-recording light as well as on the scale factor of the hologram, due to the dissimilar values of the pixel pitch in the recording camera and in the SLM array. In order to verify that such aberrations do not affect the $3 \mathrm{D}$ display, we estimated the aberration coefficients, such as coma, astigmatism, distortion, and curvature of field, that result from the reconstruction in the visible range of holograms recorded with a wavelength 20 times larger. The results show that the wavefront aberration is negligible ( $<1$ wavelength) and does not substantially affect the reconstructed images, giving the chance of a reliable IR-recording/visible-light reconstruction system [1].

Moreover, by means of a numerical procedure, we create a synthetic scene that can be optically displayed [2]. The procedure is based on recording several digital holograms of individual objects each of which rotates about its vertical axis but in a fixed position. Each holograms is geometrically transformed to change the object's position and size within a very large depth of field dispensing with the recording of holograms at different distances from the camera. In this way we obtain a dynamic 3D scene in which the statuette travels backwards and forwards in the 3D volume while performing a "pirouette". Combining holograms corresponding to different objects, it also possible to create dynamic scenes with two or more object rotating while travel back and forth.

*melania.paturzo@inoa.it; phone 39081 867-5040; fax 39081 867-5118;

O3A: Optics for Arts, Architecture, and Archaeology III, edited by Luca Pezzati, Renzo Salimbeni, Proc. of SPIE Vol. 8084, 80840K · ( ) 2011 SPIE · CCC code: 0277-786X/11/\$18 · doi: 10.1117/12.890039 


\section{METHODOLOGY}

\subsection{Experimental set-up}

The set-up for the recording and reconstruction process is shown in Fig.1. In the recording process, we use the coherent light source produced by a high power CW CO2 laser, emitting at $10.6 \mu \mathrm{m}$. The interferogram is recorded by means of an thermal imaging camera with $640 \mathrm{X} 480$ pixels with $25 \mu \mathrm{m}$ pixel pitch.

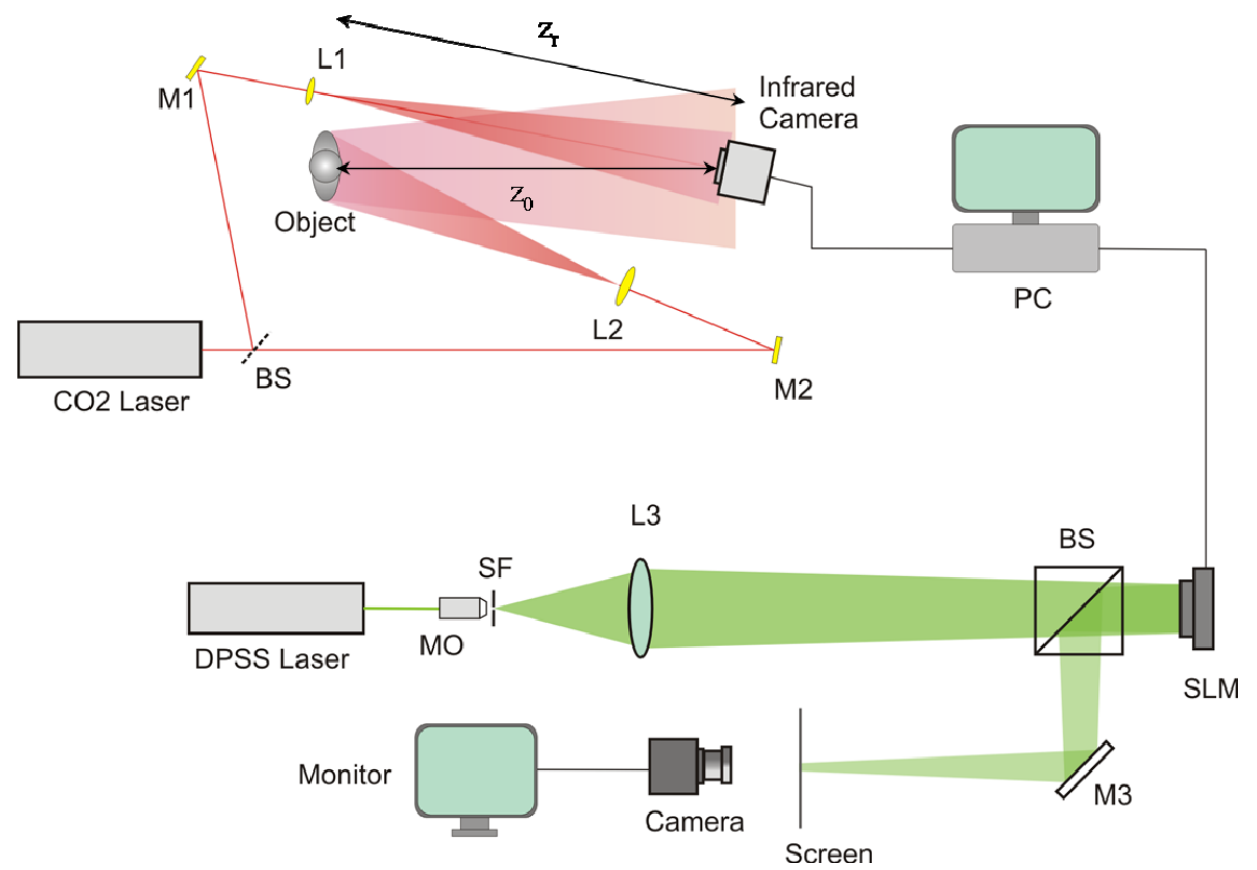

Figure 1. Experimental set-up for recording and reconstruction processes. M: mirror, L: lens, BS: beam splitter, MO: microscope objective, SLM: spatial light modulator, SF: spatial filter.

As test object we use a metallic figure: Perseus. The sculpture is a reproduction of the bronze statue by Benvenuto Cellini today at Loggia dei Lanzi in Florence, Italy. The statuette is about $33 \mathrm{~cm}$ tall. We acquire 120 holograms rotating Perseo by 3 degrees. In the reconstruction process, we use a DPSS laser emitting at $0.532 \mu \mathrm{m}$. The laser beam is expanded in such a way to obtain a convergent beam that impinges on an SLM-LCOS (PLUTO-by Holoeye) that displays the recorded hologram. The image of the statuette is produced at a fixed distance from the SLM and, then, acquired by a camera. The size of the reconstructed image at visible wavelength is about $30 \mathrm{~cm}$. In fact, we calculate the lateral magnification that in the Fourier configuration holography is expressed by the formula: $M_{\text {lat }}=\frac{1}{m} \frac{\lambda_{c}}{\lambda_{r}} \frac{z_{i}}{z_{o}}$ where $\mathrm{m}$ is scale-change factor of the hologram, given by the ratio between the pixel pitch of the SLM and that one of the recording device; $\lambda_{\mathrm{r}}$ and $\lambda_{\mathrm{c}}$ are the recording $\left(\mathrm{CO}_{2}\right.$ laser) and reconstructing (green laser $=532 \mathrm{~nm}$ ) wavelengths, respectively, while $z_{i}$ and $z_{0}$ are the distance of the image and the object by the hologram plane. To estimate the magnification we need to calculate the distance at which the reconstructed image appears in focus. The position of the 
image is obtained by the formula: $\frac{1}{z_{i}}=\frac{1}{z_{c}} \pm \frac{1}{m^{2}} \frac{\lambda_{c}}{\lambda_{r}}\left(\frac{1}{z_{0}}-\frac{1}{z_{r}}\right)$ where $\mathrm{z}_{\mathrm{r}}$ and $\mathrm{z}_{\mathrm{c}}$ are the radii of curvature of the reference beam in the recording and reconstruction process, respectively.

We note that, for a Fourier hologram $\left(\mathrm{z}_{\mathrm{o}}=\mathrm{z}_{\mathrm{r}}\right), \mathrm{z}_{\mathrm{i}}$ has the same value of the radius of curvature of the reconstructing beam, $\mathrm{z}_{\mathrm{c}}$, and does not depend on the wavelength ratio and on the scale-change factor of the hologram, $\mathrm{m}$.

\subsection{Experimental Results}

In our experiment, $\mathrm{M}_{\text {lat }}$ value is equal to 0.9 , in agreement with the measured value shown in fig.2, where the numerical reconstruction of the statue hologram and the optical reconstruction performed at a visible wavelength are illustrated.

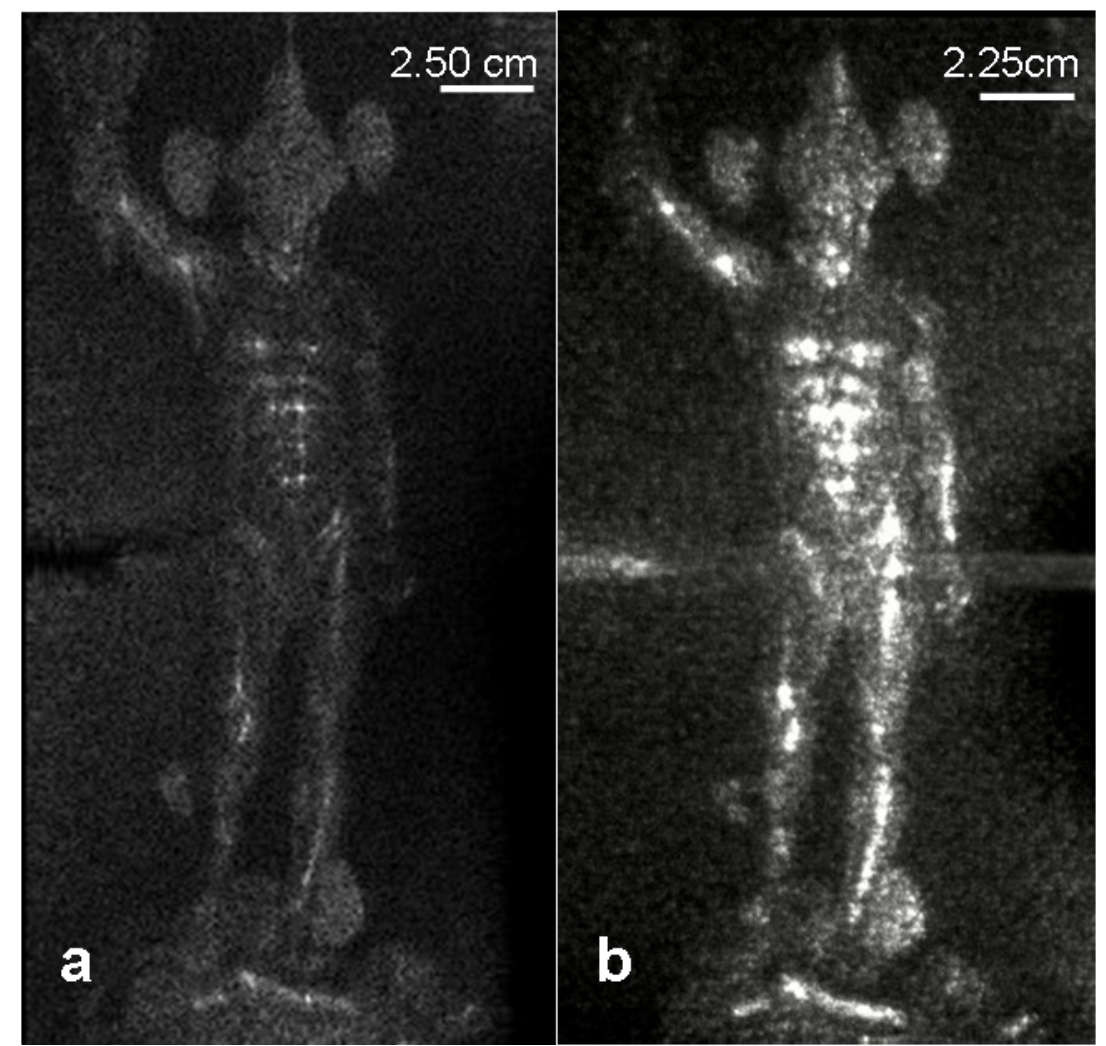

Figure 2. (a) Numerical reconstruction of the statue hologram obtained through the Fourier integral; (b) optical reconstruction performed at a visible wavelength by a monochromatic CCD camera.

Looking at the two images, no differences appear evident since the two images are scaled up so to have the same size. This implies that the following steps do not cause any distortions visible to the naked-eye: (i) hologram recording at long IR wavelength; (ii) numerical pre-processing of digital holograms (i.e. contrast optimization, noise filtering by FFT); (iii) reconstruction displayed in the visible range at 532nm; (iv) image capturing of the holographic reconstruction by a CCD camera.

To estimate the aberration coefficients, we consider the Seidel aberration formula in polar coordinates, $\rho$ and $\theta$. The spherical aberration is zero for hologram acquired in Fourier configuration, regardless of the magnitude of $z_{c}, \lambda_{r}, \lambda_{c}$ and 
$\mathrm{m}[3]$. The coma aberration is given by $W=\left(\frac{2 \pi}{\lambda_{c}}\right)\left[\frac{1}{2} \rho^{3}\left(C_{x} \cos \theta+C_{y} \sin \theta\right)\right]$ while the astigmatism is expressed by the formula $W=\left(\frac{2 \pi}{\lambda_{c}}\right)\left[-\frac{1}{2} \rho^{2}\left(A_{x} \cos ^{2} \theta+A_{y} \sin ^{2} \theta+2 A_{x y} \cos \theta \sin \theta\right)\right]$.

The field of curvature by $W=\left(\frac{2 \pi}{\lambda_{c}}\right)\left[-\frac{1}{4} \rho^{2} F\right]$ while the distortion is given by $W=\left(\frac{2 \pi}{\lambda_{c}}\right)\left[\frac{1}{2} \rho\left(D_{x} \cos \theta+D_{y} \sin \theta\right)\right]$. We calculate the aberrations coefficients exploiting the formulas in ref. 19 concerning holograms acquired in Fourier configuration. Then, considering that the lateral dimensions of SLM are such that $\rho=1.5 \mathrm{~mm}$, we obtain that the coma results about $6 / 1000 \lambda$, astigmatism $5 / 100 \lambda$, field of curvature $3 / 100 \lambda$ and distortion $4 / 10 \lambda$.

The above analysis confirms that in our experimental conditions, the wavefront aberration is negligible (lower than 1 wavelength) and, therefore, does not substantially affect the reconstructed images giving the chance for a reliable IR/recording-VIS/reconstruction holographic system.

\subsection{Synthesis of a 3D scene}

As described in ref. 2, applying a simple geometrical transformation to the hologram, the object is obtained in focus at a distance different from the original recording distance and with different lateral magnification. Based on this simple principle, we can play with all digital holograms acquired while the statuette was rotating. Each recorded hologram is geometrical transformed, by numerical stretching, to create a dynamic 3D scene in which Perseo travels backwards and forwards in the 3D volume while performing a "pirouette."

Figure 3 shows the numerical reconstruction of Perseo holograms stretched with four different elongation factors $\alpha$ ranging between 1 and 1.9. The reconstructions showed in Fig.3 are obtained using two different values of the reconstruction distance: in the first row the distance is chosen in order to have Perseus in focus when it is close-up, while in the second row it's in focus when it appears far away. When an hologram is numerically stretched, the distance at which the object will appear in focus in the reconstructed image changes. Therefore, in each row only one image appears in good focus.
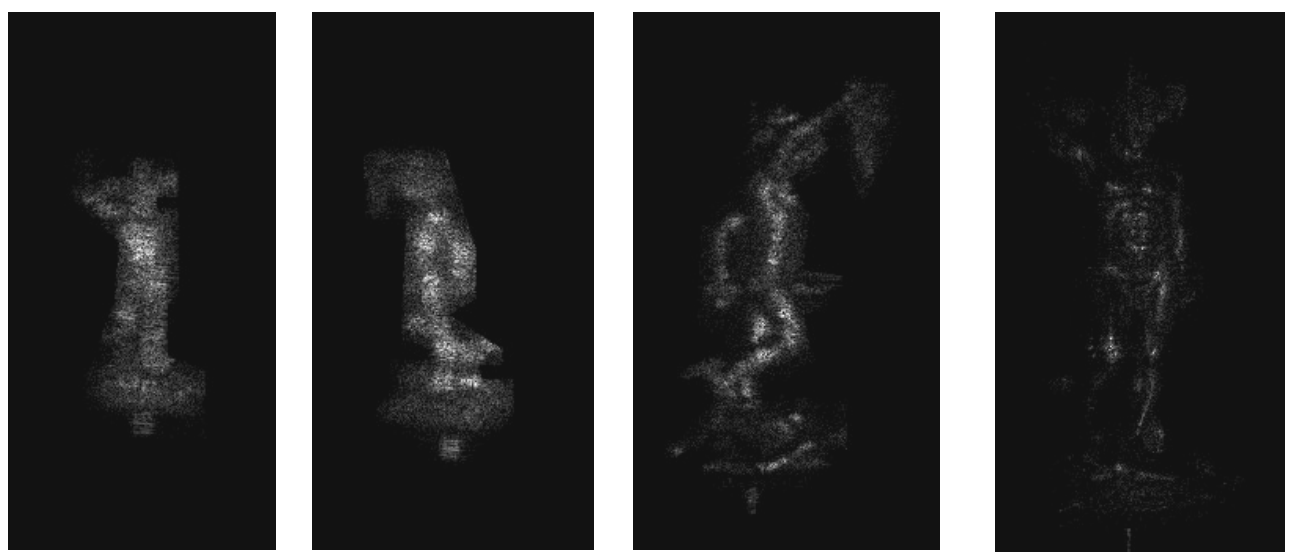

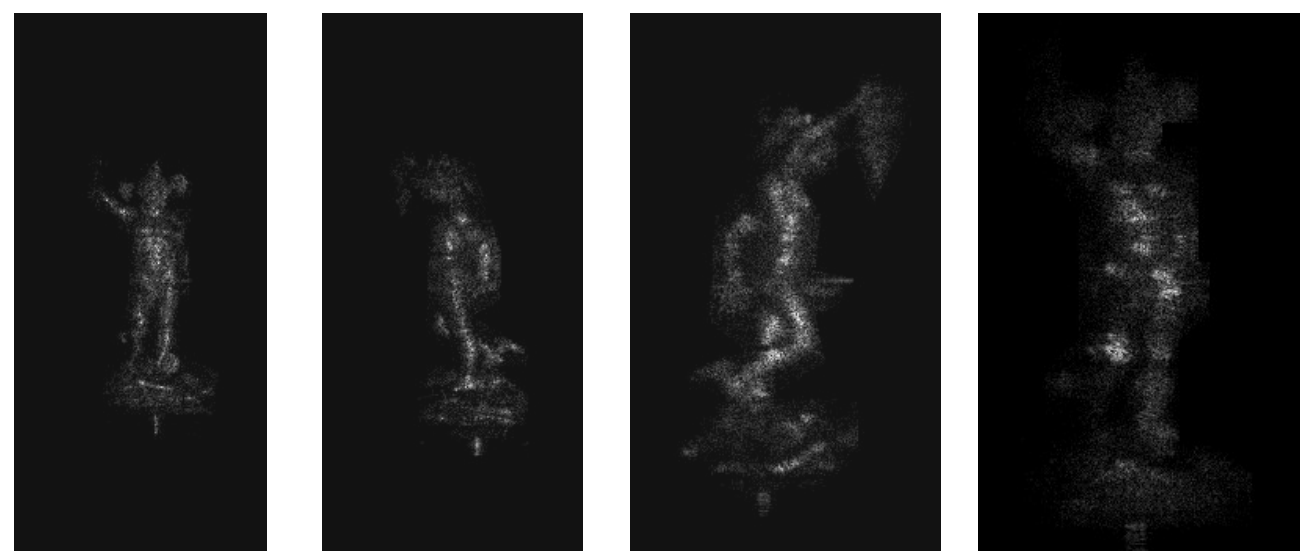

Figure 3. Numerical reconstruction of the statuette hologram stretched with different elongation factors using two different values of the reconstruction distance: the distance is chosen in order to have Perseus in focus when it is close-up (first row) or when it appears far away (second row).

It is also possible to synthesize a 3D scene by combining, coherently, digital holograms of different objects. Figure 4 shows the optical reconstruction of a synthetic hologram of Perseo and Venus obtained by the combination of two different holograms. They were recorded separately with the two statuette at the same distance and with the same optical configuration. By using the two basic original holograms and stretching them separately before combining them, we show that it is possible to synthesize a 3D scene with more than one object (see Fig. 4 and Fig.5). The position of each object in the $\mathrm{x}-\mathrm{y}$ plane is also changed by a simple numerical shift in order to avoid superimposition of the objects in the combined image. Fig.4(a,b) and Fig.5(a,b) show two numerical reconstructions, while in Fig.4c and Fig. 5c the optical reconstructions corresponding to the frame $\mathrm{b}$ are shown. In Fig.4 the distance of reconstruction is chosen in order to have Perseus \& Venus in focus when they are close-up, while in fig.5 they are in focus when they are far away.

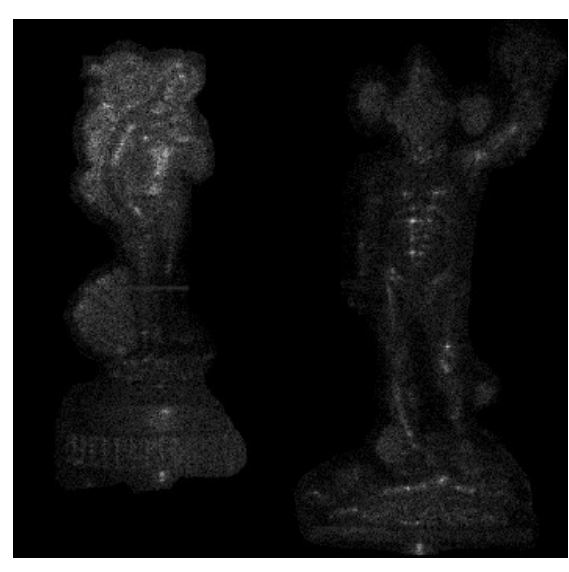

(a)

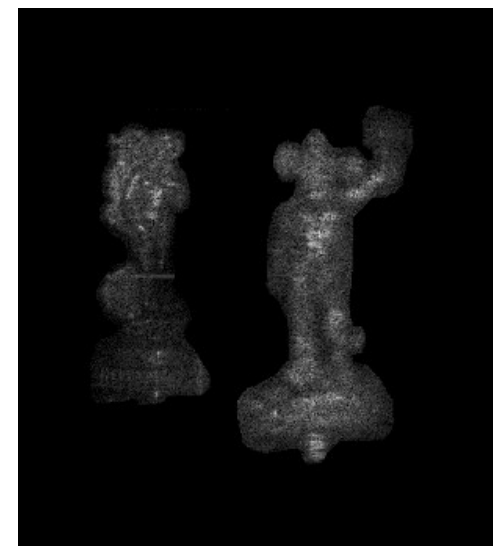

(b)

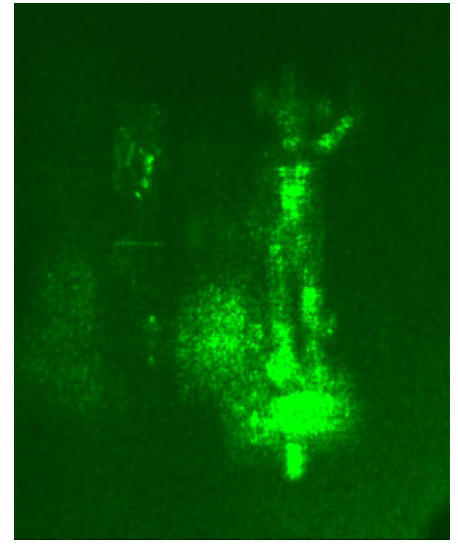

(c)

Figure 4. Numerical $(a, b)$ and optical (c) reconstructions of the synthetic holograms obtained by combining, coherently, the digital holograms of Perseus and Venus. The distance of reconstruction is chosen in order to have Perseus \& Venus in focus when they are close-up. 


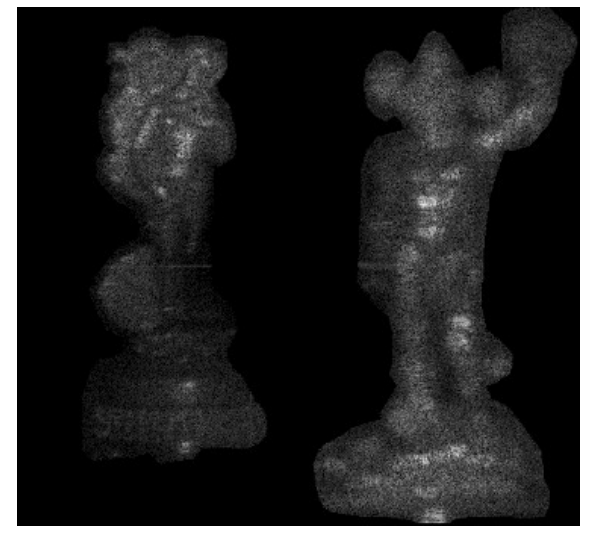

(a)

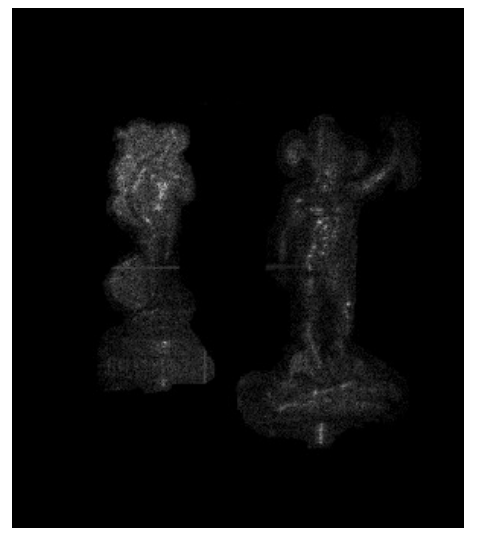

(b)

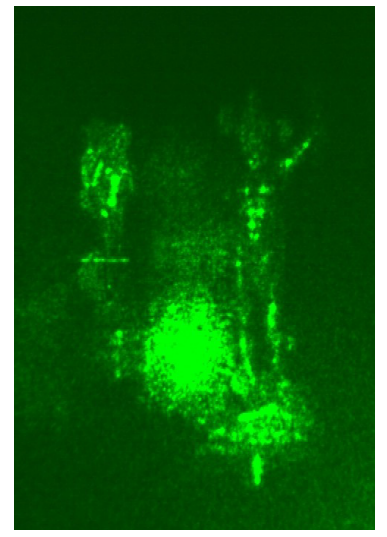

(c)

Figure 5. Numerical $(\mathrm{a}, \mathrm{b})$ and optical (c) reconstructions of the synthetic holograms obtained by combining, coherently, the digital holograms of Perseus and Venus. The distance of reconstruction is chosen in order to have Perseus \& Venus in focus when they are far away.

\section{CONCLUSION}

In conclusion, we acquired infrared digital holograms of different statuettes and combined them to create dynamic 3D scenes that, then, have been optically reconstructed by means of spatial light modulators (SLMs) using an illumination wavelength of $532 \mathrm{~nm}$.

\section{REFERENCES}

[1] Paturzo, M., Pelagotti, A., Finizio, A., Miccio, L., Locatelli, M., Gertrude, A., Poggi, P., Meucci, R., Ferraro, P., "Optical reconstruction of digital holograms recorded at $10.6 \mu \mathrm{m}$ : route for 3D imaging at long infrared wavelengths," Opt. Lett. 35, 2112-2114 (2010).

[2] Paturzo, M., Memmolo, P., Finizio, A., Näsänen, R., Naughton, T. J., Ferraro, P., "Synthesis and display of dynamic holographic 3D scenes with real-world objects," Opt. Express 18, 8806-8815 (2010).

[3] Meier, R. W., "Depth of Focus and Depth of Field in Holography," J. Opt. Soc. Am. 55, 1693-1694 (1965). 